\title{
Cloxiquine, a traditional antituberculosis agent, suppresses the growth and metastasis of melanoma cells through activation of PPAR $\gamma$
}

\author{
Wenxiang Zhang ${ }^{1,2}$, Wei Shao ${ }^{1,2}$, Zhewen Dong ${ }^{1,2}$, Shiyao Zhang ${ }^{1,2}$, Chang Liu ${ }^{1,2,3}$ and Siyu Chen ${ }^{1,2,3}$
}

\begin{abstract}
Melanoma is one of the most aggressive skin cancers and 5 -year survival rate is only $4.6 \%$ for metastatic melanoma patients. Current therapies, especially those involving clinical chemotherapy drugs, have achieved remarkable advances. However, their side effects, such as bone marrow suppression, limit the effectiveness of available pharmacological therapies. Therefore, exploring new antimelanoma drugs with less toxicity is critical for the treatment of melanoma. In the present study, we aimed to identify the antimelanoma drugs with ability to repress the proliferation of melanoma cells by using a high-content screening of FDA-approved drug libraries. We found that cloxiquine (CLQ), a traditional antituberculosic drug, exhibited strong inhibitory effects on the growth and metastasis of melanoma cells both in vivo and in vitro. In contrast, CLQ at the tested doses did not show any apparent toxicity in normal melanocytes and in the liver. At the metabolic level, treatment with CLQ decreased glycolysis, thus potentially inhibiting the "Warburg effect" in B16F10 cells. More importantly, combination of CLQ and 2-deoxyglucose (2-DG), a well-known glycolysis inhibitor, did not show a synergistic effect on the tumor growth and metastasis, indicating that inhibition of glycolysis is potentially involved in mediating CLQ's antimelanoma function. Bioinformatics analyses revealed that peroxisome proliferator-activated receptor-gamma (PPARY) served as a potential CLQ target. Mechanistically, CLQ stimulated the transcription and nuclear contents of PPARY. Furthermore, the specific PPARy inhibitor GW9662 or PPARY shRNA partially abolished the effects of CLQ. Collectively, our findings demonstrate that CLQ has a great potential in the treatment of melanoma through activation of PPARY.
\end{abstract}

\section{Introduction}

Melanoma is one of the most aggressive skin cancers, and it originates from the malignant transformation of melanocytes $^{1,2}$. Risk factors for the occurrence of melanoma include genetic susceptibility, sex, age, skin

Correspondence: Chang Liu (changliu@cpu.edu.cn) or

Siyu Chen (siyuchen@cpu.edu.cn)

${ }^{1}$ State Key Laboratory of Natural Medicines, China Pharmaceutical University,

Nanjing, Jiangsu, China

${ }^{2}$ School of Life Science and Technology, China Pharmaceutical University,

Nanjing, Jiangsu, China

Full list of author information is available at the end of the article.

Edited by S. He pigmentation, tanning ability, nevus count, freckling, and psychological health ${ }^{3,4}$. In addition, environmental features, such as occupation, latitude, and the level of ozone layer, have been identified to influence the morbidity of melanoma ${ }^{4}$. In 2017, estimated 87,110 new melanoma cases were diagnosed in the USA. This disease caused nearly 13,950 deaths, which accounts for $90 \%$ of all skin cancer-induced deaths ${ }^{5}$. Moreover, the incidence of melanoma has increased by $3.1 \%$ per year and has doubled each decade, representing a great threat to public health and entire social economies ${ }^{6}$.

\section{(c) The Author(s) 2019}

(c) (i) Open Access This article is licensed under a Creative Commons Attribution 4.0 International License, which permits use, sharing, adaptation, distribution and reproduction c. in any medium or format, as long as you give appropriate credit to the original author(s) and the source, provide a link to the Creative Commons license, and indicate if changes were made. The images or other third party material in this article are included in the article's Creative Commons license, unless indicated otherwise in a credit line to the material. If material is not included in the article's Creative Commons license and your intended use is not permitted by statutory regulation or exceeds the permitted use, you will need to obtain permission directly from the copyright holder. To view a copy of this license, visit http://creativecommons.org/licenses/by/4.0/. 
The proliferation and metastasis of malignant cells are the major underlying biological processes of melanoma cancer. Although clinical chemotherapy drugs, including cisplatin and nitrogen mustard, antagonize melanoma progression, their toxic side effects, such as bone marrow and immune suppression, limit the effectiveness of currently available pharmacological therapies ${ }^{7-9}$. On the other hand, with advances in the genotype-directed treatment, specific and highly effective small-molecule inhibitors targeting BRAF and MEK mutants have been developed and used in the treatment of BRAF-mutant metastatic melanoma patients. However, drug resistance occurs and renders these drugs ineffective ${ }^{10}$. Similarly, new immunotherapeutic strategies, such as using antibodies against cytotoxic T-lymphocyte-associated antigen 4 (CTLA4, ipilimumab) and programmed cell death 1 (PD-1) or programmed death ligand 1 (PD-L1, pembrolizumab or nivolumab), are beneficial to only a subset of the patients in clinics ${ }^{11,12}$. Therefore, a new strategy aiming to suppress melanoma progression may have a high value in the therapy of melanoma cancer.

Given that the de novo development of new drugs is a time-consuming and costly process, drug reposition serves as an effective and innovative approach to hasten the development cycles, especially those in cancer therapeutics. More importantly, repositioned drugs have the minimal risk in the clinical uses due to the comprehensive pharmacological, safety and toxicological data ${ }^{13}$. For instance, as an anti-diabetic drug, metformin decreases human cancer incidence, thus improving the survival of cancer patients ${ }^{14}$. Therefore, we focused on drug reposition and presented a CCK-8 assay-based drug screening in mouse B16F10 melanoma cells using the FDA-approved drug library (composed of 1430 small compounds). Among these, we found that cloxiquine (CLQ) exhibited strong antimelanoma properties. CLQ is a well-known anti-infection and antibacterial drug clinically used in the treatment of tuberculosis. Notably, CLQ has a unique p53-modulating activity that shifts its transactivation from pro-apoptotic to protective responses, including enhancing p21 induction, thereby protecting mice from $\gamma$-irradiation-induced gastrointestinal death ${ }^{15}$. In addition, CLQ protects from cardiac ischemia-reperfusion (IR) injury in mice by uncoupling mitochondria and inducing autophagy $^{16}$. However, the beneficial action of CLQ on melanoma and the direct molecular target through which CLQ exerts its antitumor function remain unknown. In our present study, we demonstrated that CLQ exerted strong antimelanoma properties in vitro and in vivo, and the antitumor effects of CLQ were partially, if not totally, mediated by peroxisome proliferator-activated receptorgamma (PPARy). Collectively, our findings offer new perspectives for the potential of CLQ in melanoma treatment.

\section{Results \\ Compound screenings identify CLQ as a potential antimelanoma drug}

To identify novel inhibitors of melanoma cell growth, 1430 small compounds ( $10 \mu \mathrm{M}$ final concentration) from the FDA-approved drug library were screened for their ability to repress cell proliferation of mouse B16F10 cells (Fig. 1a). CCK- 8 analysis indicated that 27 drugs exhibited dramatic inhibitory effects on the viability of these cells (inhibition ratio $>75 \%$ ). For the future translational medicine purpose, we double checked the actions of these drugs on human A375 melanoma cells and found that 19 of which demonstrated similar functions (Fig. 1b). Among them, 18 drugs are already known as antitumor agents or their targets are involved in tumorigenesis (Supplementary Table 1), and only CLQ (Fig. 1c), a classic antituberculosis drug, has never been reported to be functional for the cancer therapy. Therefore, it is interesting to explore potential antimelanoma effects of CLQ in the present study. Importantly, although CLQ suppressed both B16F10 and A375 cell growth in a dosedependent manner, it did not affect the viability of normal Melan-A and PIG1 melanocytes, suggesting that it is safe for non-tumor cells (Fig. 1d, e). These results were confirmed by morphological observations (Fig. 1f).

\section{CLQ inhibits the proliferation of melanoma cells and suppresses tumor growth in a mouse B16F10 melanoma xenograft model}

To further determine the antiproliferative actions of CLQ on melanoma cells, we performed an EdU incorporation assay. We found a significant dose-dependent reduction in cell proliferation after 24-hour (h) CLQ treatment. CLQ at the dose of $2.5 \mu \mathrm{M}$ or $10 \mu \mathrm{M}$ exhibited the strongest inhibitory effects on the B16F10 cells (inhibition by 73\%) or the A375 cells (inhibition by 64\%), respectively (Fig. 2a and Supplementary Fig. 1). At the molecular level, CLQ reduced the protein expression of PCNA, a hallmark of cell proliferation (Fig. 2b). As is known, activated cell proliferation is tightly associated with accelerated cell cycle progression, which is interlocked by the coordination between positive regulators CDK/Cyclin complex and negative regulators of the CDKN1 family (e.g., p27 and p21) ${ }^{17}$. Coincided with this principle, we found that CLQ decreased the protein expression levels of Cyclin E, Cyclin D1, CDK4 and CDK2 in a concentration-dependent manner. In contrast, the protein levels of p27 and p21 were correspondingly increased by CLQ treatment (Fig. 2b, c). Next, we used B16F10 tumor xenograft models to evaluate the suppressive effect of CLQ in vivo. As shown in Fig. 2d, the average tumor volume in the control group reached $5212.63 \mathrm{~mm}^{3}$, whereas $5 \mathrm{mg} / \mathrm{kg}$ and $25 \mathrm{mg} / \mathrm{kg}$ CLQ treatments remarkably decreased tumor volume by 66.37 


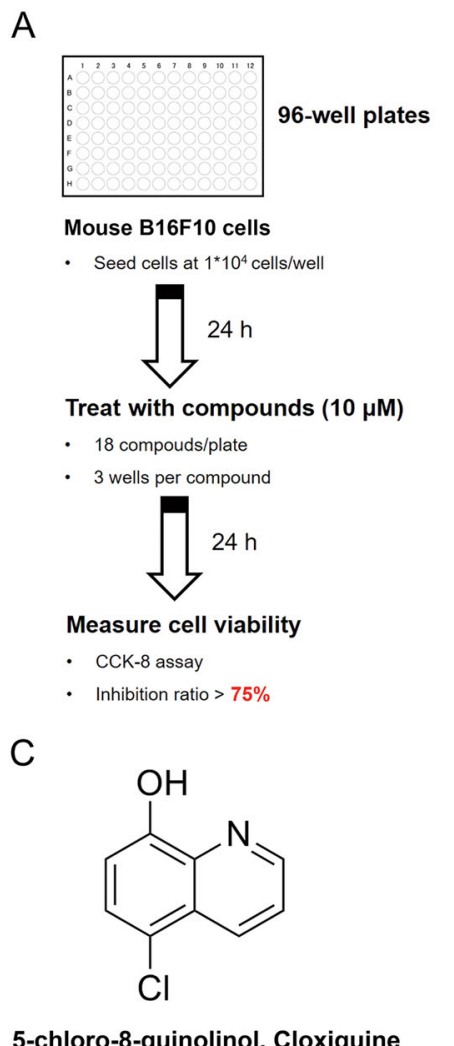

5-chloro-8-quinolinol, Cloxiquine

D $\square \mathrm{CTL}$
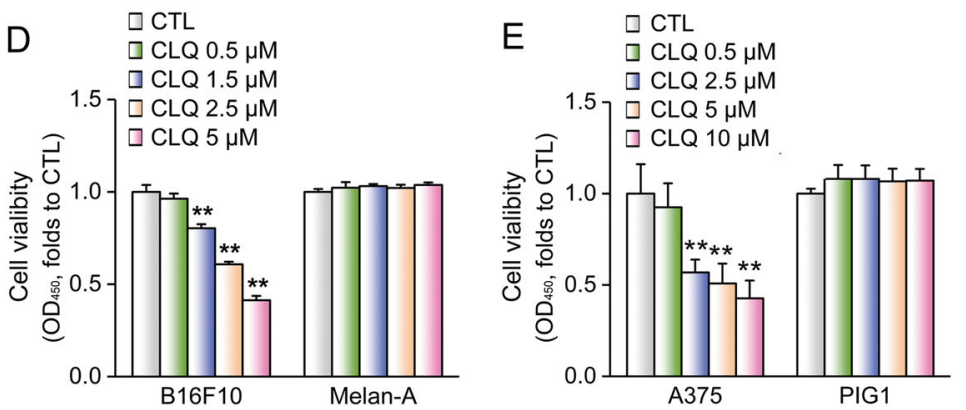

F

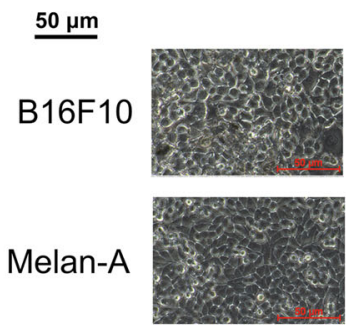

CTL

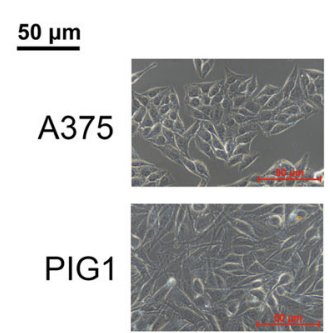

CTL
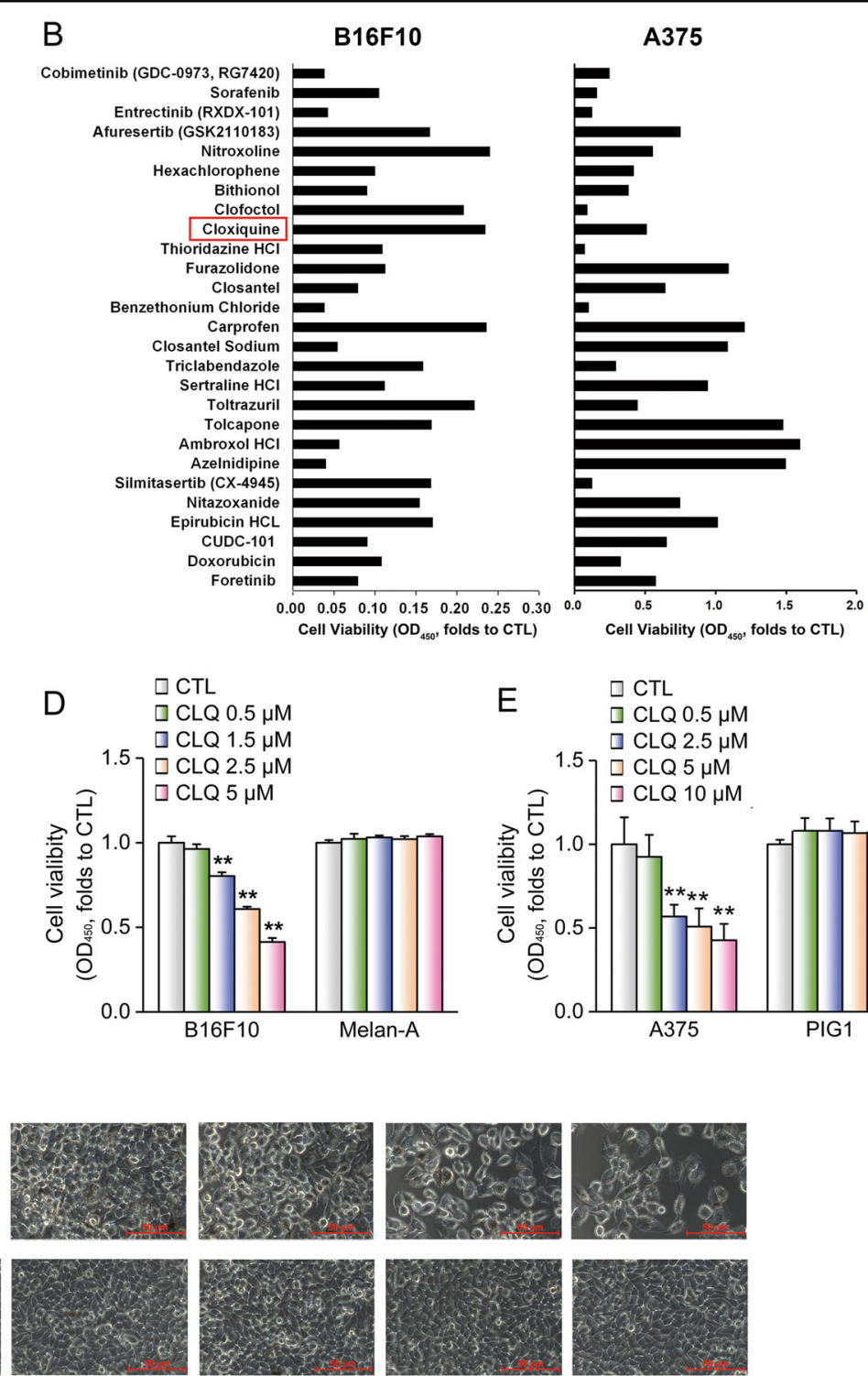

2.5

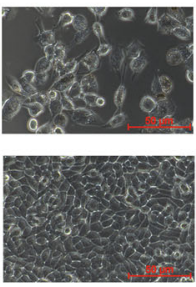

1.5

5

CLQ $(\mu \mathrm{M})$

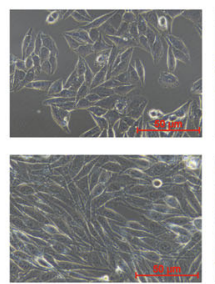

0.5
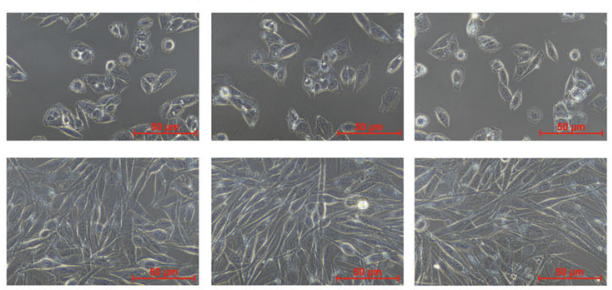

2.5

5

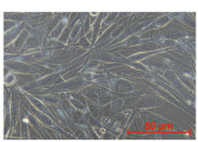

$\mathrm{CLQ}(\mu \mathrm{M})$

Fig. 1 Compound screenings in B16F10 cells identify CLQ as a potential antimelanoma drug. a The schematic workflow of the compound screen. b Cell viability of melanoma (B16F10 and A375) cells treated with indicated functional drugs. c Chemical structure of CLQ. d, e Mouse B16F10 and Melan-A melanocyte cells, as well as the human A375 and PIG1 cells were treated with the indicated concentrations of CLQ for $24 \mathrm{~h}$. The inhibitory effect of CLQ on cell viability was detected by a CCK-8 assay. $\mathbf{f}$ Morphological analysis. All values are presented as the mean \pm SD from at least three separate experiments. ${ }^{* *} P<0.01$ vs. CTL 
A

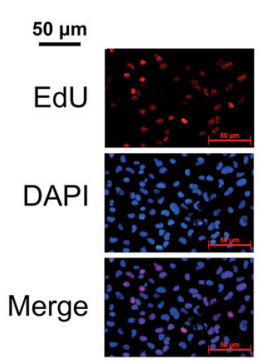

CTL

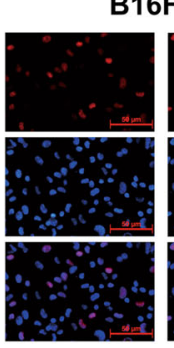

0.5
16F10

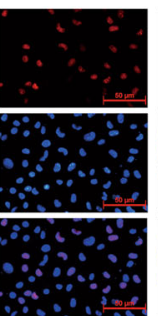

1.5

$\mathrm{CLQ}(\mu \mathrm{M})$

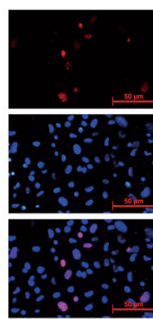

2.5
B

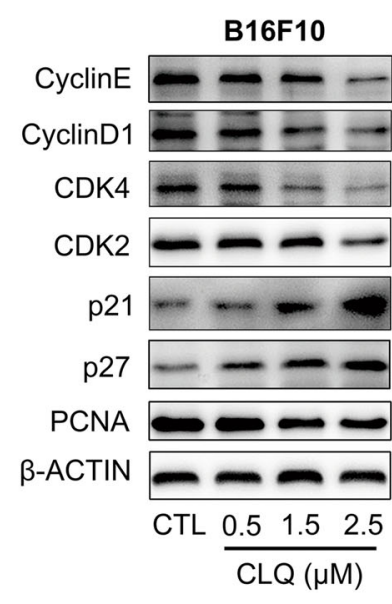

D

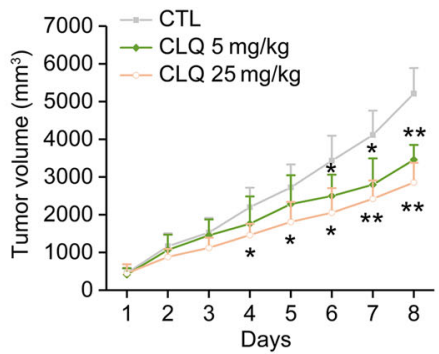

G

$50 \mu \mathrm{m}$

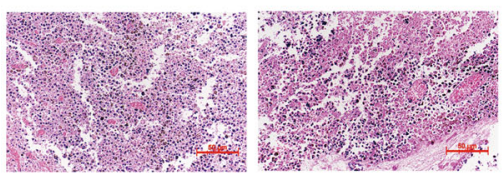

CTL
H\&E staining

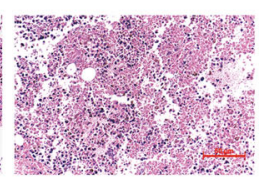

A375

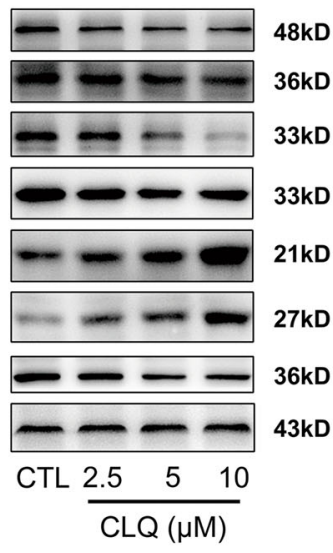

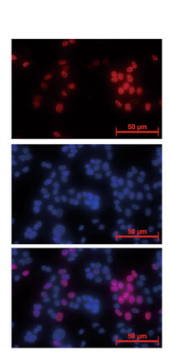

CTL

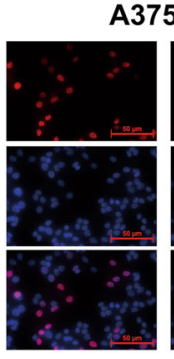

2.5

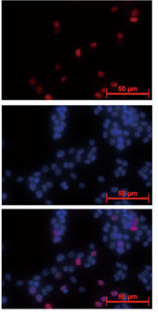

5

CLQ $(\mu \mathrm{M})$

C
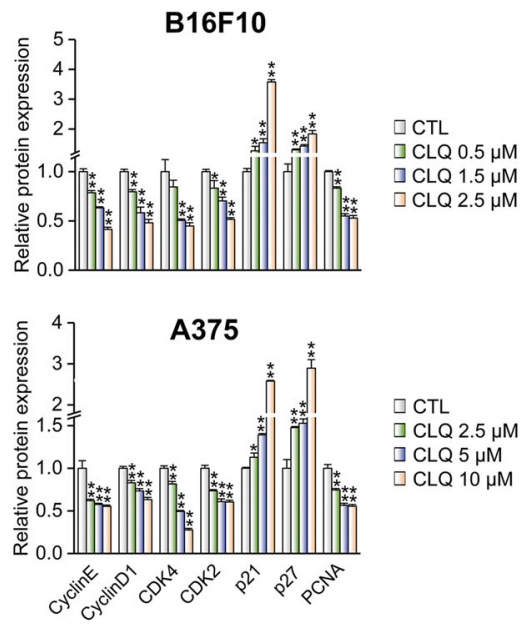

$\square \mathrm{CTL}$

$\square \mathrm{CLQ} 2.5 \mu \mathrm{M}$

1 $\mathrm{CLQ} 5 \mu \mathrm{M}$

$\square \mathrm{CLQ} 10 \mu \mathrm{M}$
E

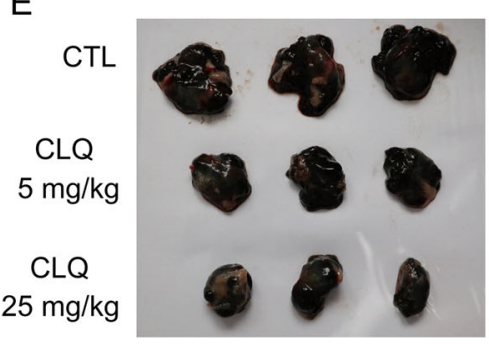

F

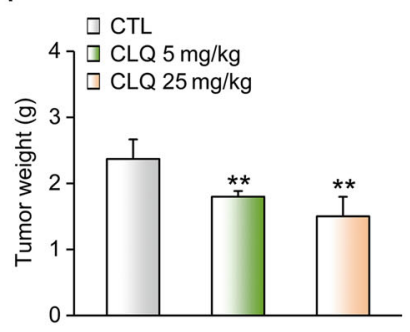

Fig. 2 CLQ inhibits the proliferation of melanoma cells and suppresses tumor growth in vivo. B16F10 and A375 cells were treated with the indicated concentrations of CLQ for $24 \mathrm{~h}$. a EdU incorporation assay used to measure cell proliferation. $\mathbf{b}$ Protein expression levels of key regulators involved in cell cycle progression. c Quantification for the immunoblots from $\mathbf{b}$. All values are presented as the mean \pm SD from at least three separate experiments. ${ }^{*} P<0.05$ and ${ }^{*} P<0.01$ vs. CTL. B16F10 cells $\left(1 \times 10^{6}\right.$ cells per mouse) were injected subcutaneously into the right flank of nude mice. When tumors became palpable on day 7 , mice were randomly divided into three groups and were administered either vehicle (olive, i.p., daily) or CLQ ( $5 \mathrm{mg} / \mathrm{kg}, 25 \mathrm{mg} / \mathrm{kg}$, i.p., daily) for 8 days. $n=5$ for each group. $\mathbf{d}$ Subcutaneous tumor volumes were measured at the indicated days. e Representative tumor images. $\mathbf{f}$ Tumor weights. $\mathbf{g}$ H\&E staining and immunohistochemistry analysis of Ki-67 from tumor sections. All values are presented as the mean $\pm \mathrm{SD}$. ${ }^{*} P<0.05$ and ${ }^{* *} P<0.01$ vs. $\mathrm{CTL}$ 
and $54.79 \%$, respectively, when compared to the control group. Consistently, the xenograft tumor weights were reduced by 75.91 and $63.41 \%$ (Fig. 2e, f). Histologically, hematoxylin and eosin $(\mathrm{H} \& \mathrm{E})$ staining analysis showed that the tumors were composed of densely packed cells from the control group, whereas the administration of CLQ significantly decreased the tumor cell density and blurred tumor cell borders. Immunohistochemistry (IHC) analysis indicated that the number of Ki-67-positive cells gradually reduced in response to CLQ administration (Fig. $2 \mathrm{~g})$. In contrast, we did not observe any hepatotoxicity in CLQ-treated mice, as evidenced by modest alterations in serum alanine transaminase (ALT) and aspartate transaminase (AST) levels (Supplementary Fig. 2a). Coincided with these results, we found that the morphology of liver cells was clear and the intracellular margin was discernable, as revealed by H\&E staining. At the meanwhile, terminal-deoxynucleoitidyl transferase mediated nick end labeling (TUNEL) assays showed barely DNA fragmentation in the liver sections of these mice (Supplementary Fig. 2b, c). Both these staining results suggest that CLQ is quite safe when administered in vivo.

\section{CLQ inhibits the migration of melanoma cells and} suppresses tumor metastasis in mouse B16F10 melanoma lung metastatic model

Similar to the proliferation results, CLQ decreased the migration rates of B16F10 and A375 cells when assessed by transwell chamber and wound-healing assays (Fig. 3a). The quantitative results were presented in Supplementary Fig. 3. At the molecular level, the expression levels of migration-associated proteins, including ICAM-1, VCAM-1, MMP-9 and MMP-2, were blocked by treatment with CLQ in a dose-dependent manner (Fig. 3b, c). Consistently, by using a B16F10 melanoma metastasis mouse model, we found the number of macroscopic black metastases on the surfaces of the lungs was decreased in the CLQ-treated group when compared with the control group (Fig. 3d, e). H\&E staining analysis in lungs showed that the gross morphology of B16F10 melanoma metastasis was alleviated by the administration of CLQ for 14 days (Fig. 3f).

\section{CLQ suppresses glycolysis in melanoma cells}

By undergoing aerobic glycolysis (also known as Warburg effect), cancer cells consume glucose to produce lactate, which is the final product of glycolysis, thus providing them with a growth advantage ${ }^{18,19}$. We then investigated if CLQ-inhibited melanoma cell proliferation and migration by blocking glycolysis. As shown in Fig. 4a-c, CLQ increased glucose levels in the culture medium and decreased lactate production, as well as ATP generation, in B16F10 cells. Furthermore, CLQ-treated B16F10 cells exhibited decreased extracellular acidification rate (ECAR), which is an indicator of an overall glycolytic flux (Fig. 4d and Supplementary Fig. 4a). Accordingly, we found a remarkable reduction in the mRNA and protein expression levels of glucose transporter 1 (GLUT1), hexokinase-2 (HK2), pyruvate kinase M2 (PKM2) and lactate dehydrogenase (LDHA), in B16F10 cells after 24-h exposure to CLQ at the indicated doses (Fig. 4e, f and Supplementary Fig. 4b).

\section{Combination of 2-DG and CLQ does not exhibit synergistic antimelanoma effects}

To dissect the role of glycolysis in CLQ-induced impairment of B16F10 growth and metastasis, we treated the cells with either CLQ, 2-deoxyglucose (2-DG, which is a glucose analog and blocks the first step of glycolysis), or their combination. As shown in Supplementary Fig. 5a, while 2-DG and CLQ alone repressed the proliferation and migration of B16F10 cells, the combination of these two reagents did not lead to a further inhibition. The quantitative results were presented in Fig. 5 b. Consistently, the protein expression levels associated with accelerated proliferation and migration were reduced, whereas the negative cell cycle regulators p27 and $\mathrm{p} 21$ were induced by 2-DG and CLQ (Supplementary Fig. 5c, d). Consistent with in vitro observations, combination of 2-DG and CLQ treatments did not exhibit a synergistic effect on the suppression of tumor growth and metastasis in vivo, compared with 2-DG or CLQ treatment alone (Supplementary Fig. 5e-1).

\section{Bioinformatics analyses identifies PPARY as a potential drug target of CLQ}

While it has been reported that CLQ has a unique p53modulating activity and it increases p53 protein expression in human T-cell leukemia MOLT-4 cells, p53 protein levels were modestly regulated upon CLQ treatment in our study (Supplementary Fig. 6a), suggesting that other molecular targets mediate antimelanoma effects of CLQ. To address this concern, we performed bioinformatic analyses by using PharmMapper and DRAR-CPI software. A cluster of the top 10 candidates revealed that PPARY was a potential CLQ target protein (Fig. 5a and Supplementary Tables 2 and 3). To confirm this result, RT-qPCR and western blot analyses were performed to detect the regulation of CLQ in PPARy actions in B16F10 cells. Time-course analysis indicated that both PPAR $\gamma$ mRNA and protein expression levels were increased by CLQ in a time-dependent manner up to 12-h treatment and then gradually decreased to the basal level at $24 \mathrm{~h}$ (Fig. 5b, c and Supplementary Fig. 6b). Notably, increase of PPAR $\gamma$ nuclear contents represents as a hallmark of PPARY activation in addition to its expression. Thus, we performed immunoblot analyses and immunofluorescence (ICC) in cellular fractions of B16F10 cells. We found that 


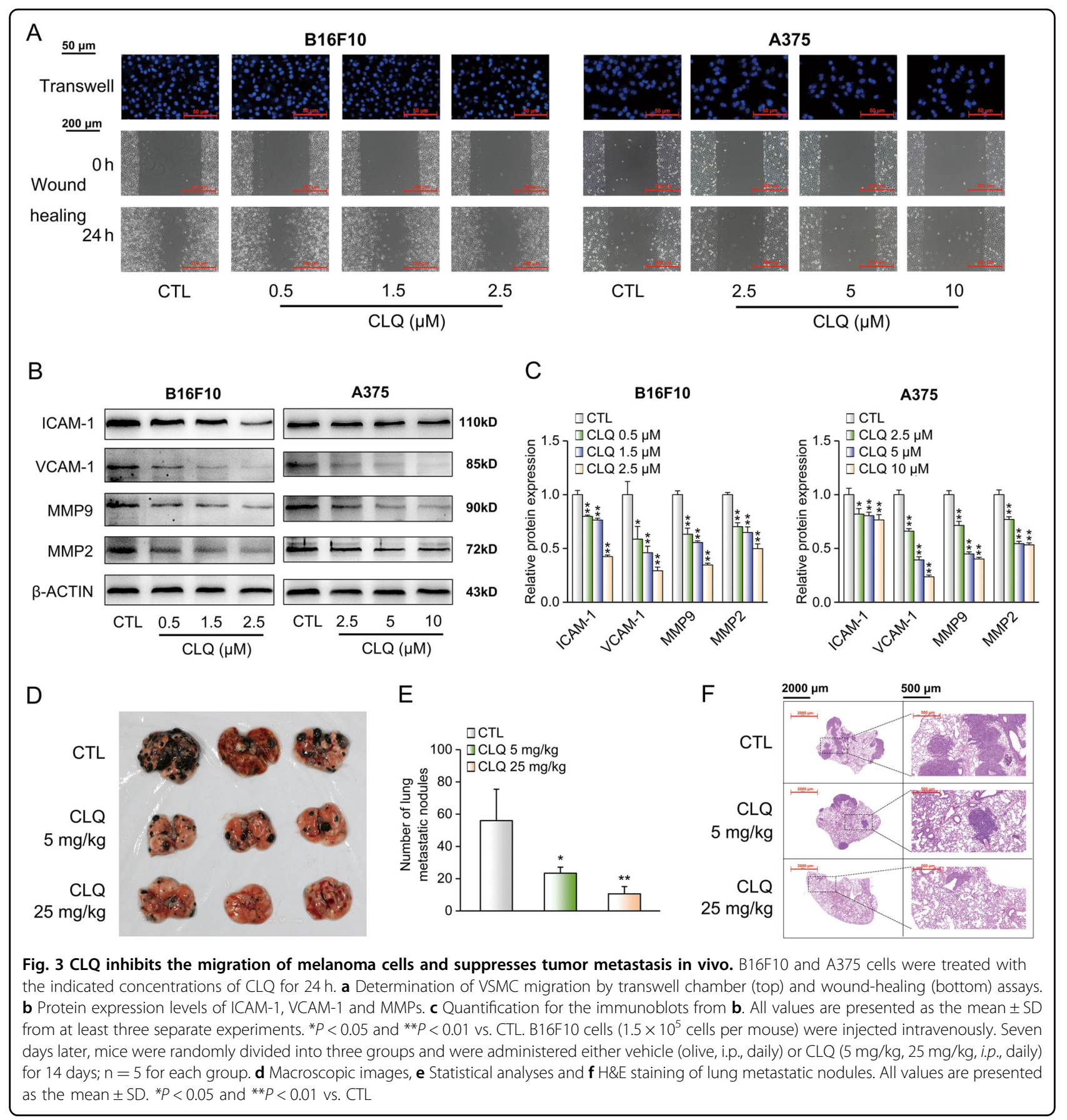

treatment with CLQ led to an increase of PPARy both in the cytosol and in nucleus (Fig. 5d-f and Supplementary Fig. 6c), implicating the activation of PPARY induced by CLQ.

\section{PPARY mediates the antimelanoma effects of CLQ in B16F10 cells}

To explore the causal relation between PPAR $\gamma$ activation and CLQ pharmalogical effects, we treated B16F10 cells with GW9662 (known as a PPAR $\gamma$-specific inhibitor) and PPARy shRNA (knockdown efficiency was presented in Supplementary Fig. 7), respectively. As shown in Fig. $6 \mathrm{a}$, the CLQ-induced reduction in cell proliferation was partially attenuated by GW9662. The quantitative results were presented in Supplementary Fig. 8a. Accordingly, the inhibition of the protein expression levels of PCNA, Cyclin D1 and CDK4 in CLQ-treated cells was significantly abolished, whereas the accumulation of negative cell cycle regulators, including p27 and p21, was decreased in GW9662/CLQ-cotreated cells (Fig. 6b and 


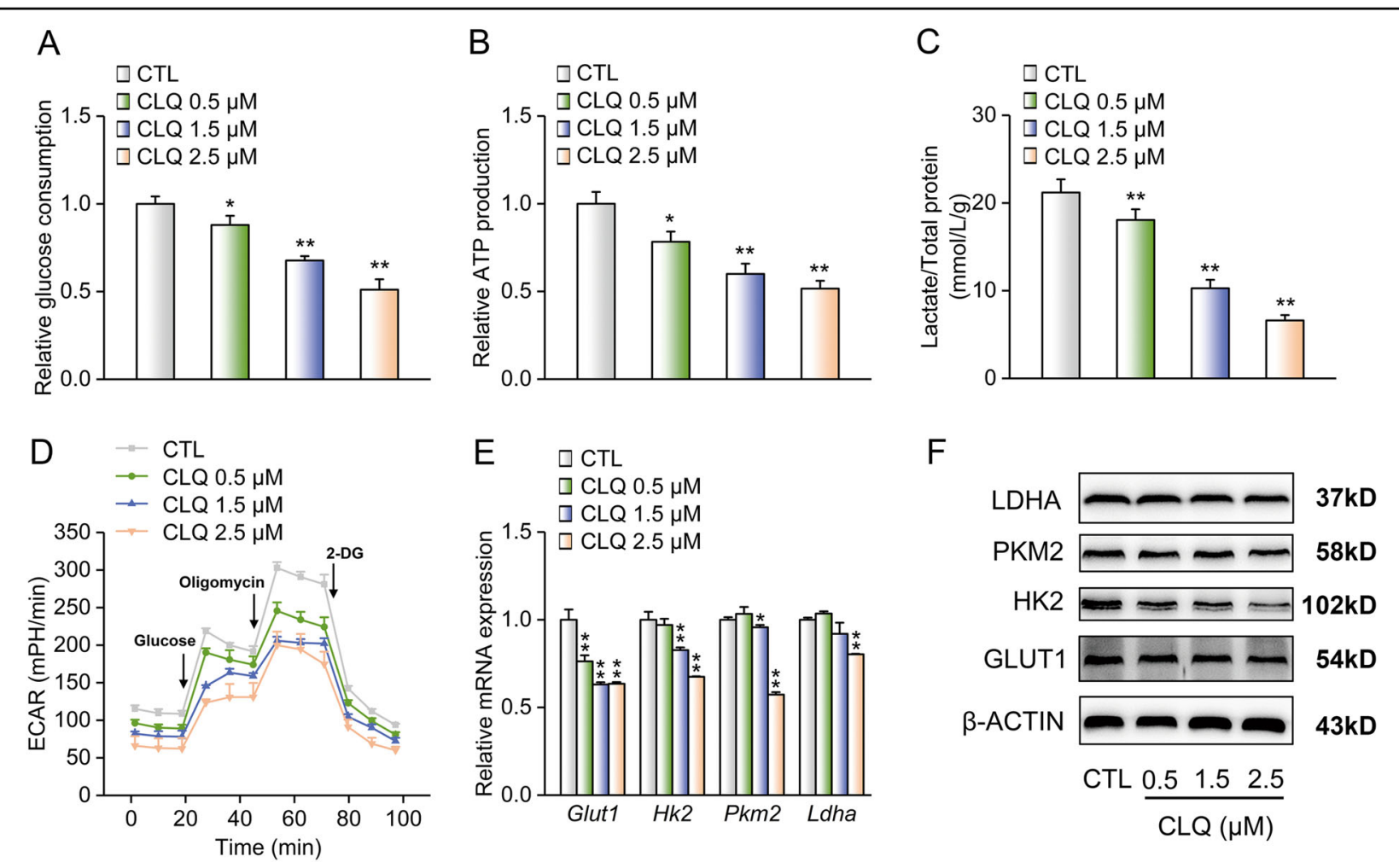

Fig. 4 CLQ suppresses the glycolysis in melanoma cells. Mouse B16F10 cells were treated with the indicated concentrations of CLQ for $24 \mathrm{~h}$. a Glucose consumption. b Relative ATP production. $\mathbf{c}$ Lactate production. $\mathbf{d}$ ECAR. e RT-qPCR and $\mathbf{f}$ Western blot analyses of GLUT1, HK2, PKM2 and LDHA expression levels. All values are presented as the mean \pm SD from at least three separate experiments. ${ }^{*} P<0.05$ and ${ }^{*} P<0.01$ vs. CTL

Supplementary Fig. 8b). For cell migration, woundhealing assay indicated that GW9662 antagonized the anti-migration properties of CLQ (Fig. 6c). The quantitative results were presented in Supplementary Fig. 8c. At the molecular level, CLQ-induced suppression of the migration-related proteins, such as VCAM-1 and MMP-9, was strongly alleviated by GW9662 (Fig. 6d and Supplementary Fig. 8d). Similar results were observed when PPARy was specifically knocked down by shRNA, emphasizing a critical role of PPAR $\gamma$ in mediating the antimelanoma action of CLQ (Supplementary Fig. 9).

\section{PPAR y mediates the inhibitory effects of CLQ on glycolysis in B16F10 cells}

To determine the mediatory role of PPAR $\gamma$ in CLQsuppressed glycolysis, we similarly incubated B16F10 cells with GW9662. As expected, we found that GW9662 significantly accelerated glucose consumption while increasing lactate production and ATP generation in CLQ-treated B16F10 cells (Fig. 7a-c). Consistently, ECAR analysis indicated that the CLQ-inhibited glycolytic flux was abolished in presence of GW9662 (Fig. 7d and Supplementary Fig. 10a). At the molecular level, GW9662 abrogated the CLQ-induced reduction of glycolytic genes, including $\mathrm{HK} 2, \mathrm{PKM} 2$ and LDHA, at both the transcriptional and translational levels (Fig. 7e, $\mathrm{f}$ and Supplementary Fig. 10b).

\section{Discussion}

In our present study, we performed cellular screening with the FDA-approved drug library to identify new suppressors of melanoma. CLQ was ultimately selected for further characterization as an antimelanoma drug not only because of its strong inhibitory effects on B16F10 cell proliferation (inhibition rate $>75 \%$ ) but also because its antitumor effect has never been demonstrated before. We found that CLQ decreased melanoma tumor growth and metastasis in vivo and in vitro. In addition, CLQ ameliorated the "Warburg effect" in melanoma B16F10 cells. On the other hand, PharmMapper and DRAR-CPI analyses indicated that PPAR $\gamma$ was the most likely molecular target in relaying CLQ signals. More importantly, the specific PPAR $\gamma$ inhibitor GW9662 or its shRNA partially abolished antimelanoma effects of CLQ.

As a well-established drug, CLQ is used to treat tuberculosis and exhibits powerful anti-infection and antibacterial properties ${ }^{20}$. A maximum tolerated dose study in mice revealed that the administration of CLQ (dosages $<80 \mathrm{mg} / \mathrm{kg}$ ) did not lead to any overt signs of toxicity, including abnormal behavior or other side 
A

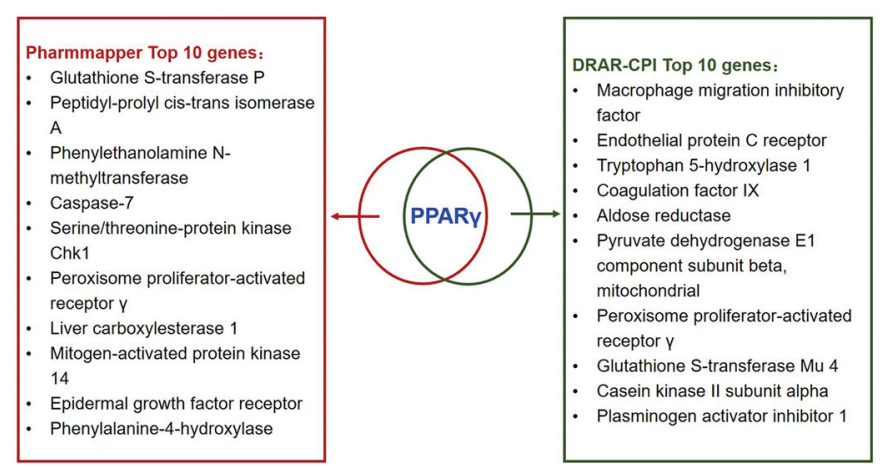

D

\section{Cytosol}
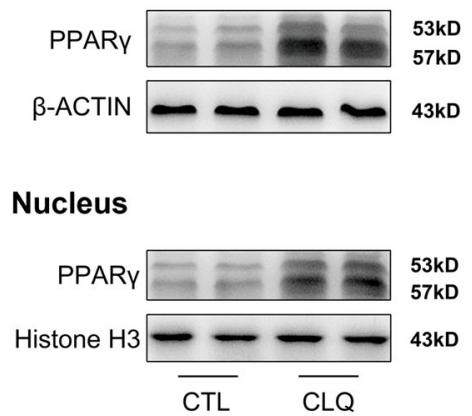

B

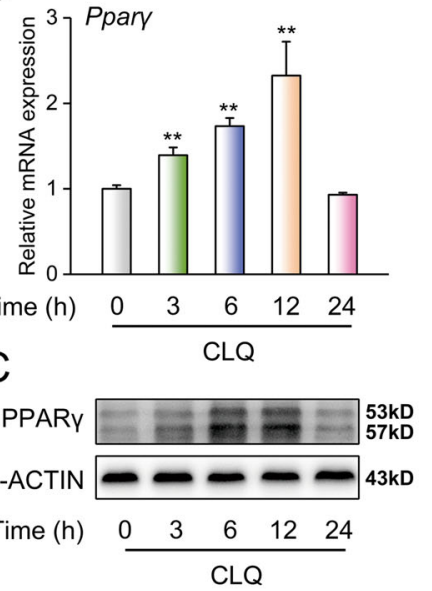

F
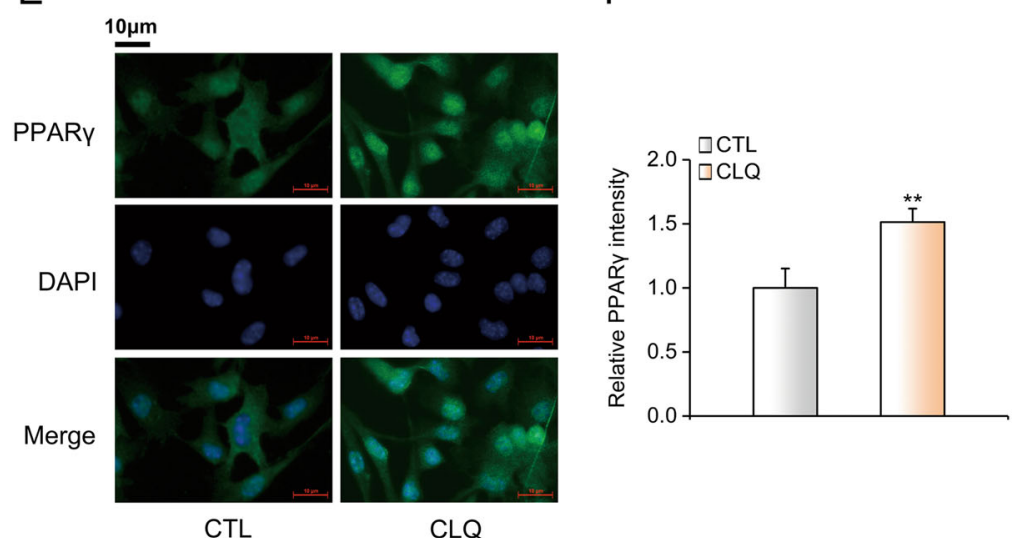

Fig. 5 Bioinformatic analyses identify PPARy as a potential drug target of CLQ. a A cluster of the top 10 candidates from bioinformatics analyses using PharmMapper and DRAR-CPI software. To investigate the effect of CLQ on PPARy expression, B16F10 cells were treated with $1.5 \mu \mathrm{M}$ CLQ for the indicated time-points. b RT-qPCR and (c) Western blot analyses of total PPARy expression levels. ${ }^{* *} P<0.01$ vs. $0 \mathrm{~h}$. B16F10 cells were treated with $1.5 \mu \mathrm{M} \mathrm{CLQ}$ for $12 \mathrm{~h}$. d Protein levels of cytosolic and nuclear PPARy expression. e Immunofluorescence analysis of PPARY protein expression in B16F10 cells. $\mathbf{f}$ Quantitative data of (e). All values are presented as the mean \pm SD from at least three separate experiments. ${ }^{* *} P<0.01$ vs. CTL

effects $^{15}$. Therefore, the doses we used (maximum dose $25 \mathrm{mg} / \mathrm{kg}$ ) in mice are rather low and considered safe. We also evaluated key parameters involved in the assessment of liver toxicity, including the serum AST and ALT levels, liver histology and hepatocytes apoptosis. As shown in Supplementary Fig. 2, all these parameters were within their physiological ranges. Therefore, such a low dose of CLQ may dramatically decrease toxic crisis and improve patient tolerance in the future translational applications. More studies are needed to pursue the antimelanoma effects of CLQ in the clinic based on these low doses.

The overactivated growth of melanoma cells is an important event in the pathogenesis of cancer and is directly linked to the failure of clinical interventions used to treat melanoma cancer patients. In the present study, CLQ significantly decreased proliferation of melanoma cells and B16F10 xenograft tumor growth. In addition, the cell cycle is tightly related to cell proliferation and is finely controlled by multiple proteins, such as CDKs, cyclins and CDK inhibitors $^{21}$. Our analyses showed a dramatic dosedependent induction of the CDK inhibitors p21 and p27 and a marked reduction of CDKs (CDK2 and CDK4) and cyclins (Cyclin E and Cyclin D1) in CLQ-treated B16F10 and A375 cells. In addition, tumor metastasis represents a major complication in clinical treatment lowering the survival rate of melanoma cancer patients ${ }^{22}$. In our study, we found that CLQ exhibited an excellent ability to inhibit migration of melanoma cells in vitro and remarkably decreased lung metastasis of B16F10 in vivo. Additionally, the protein expression levels of metastasis-associated genes (such as MMP2/9, ICAM-1 and VCAM-1) in melanoma cells were suppressed by CLQ treatment. 
A

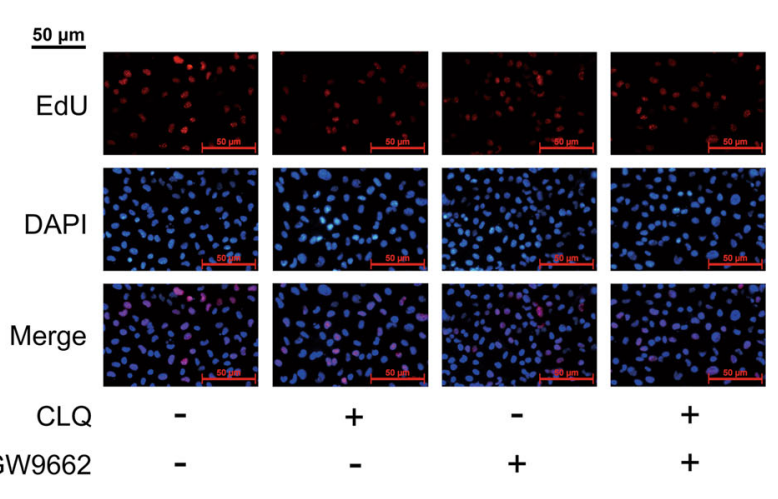

C

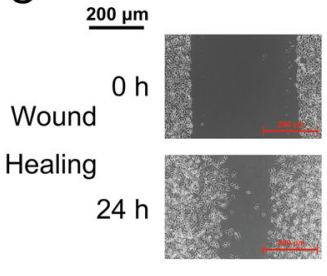

CLQ

GW9662

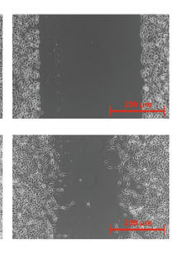

$+$

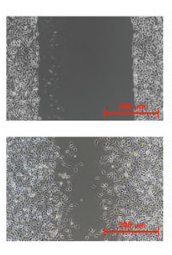

$-$

$+$
B

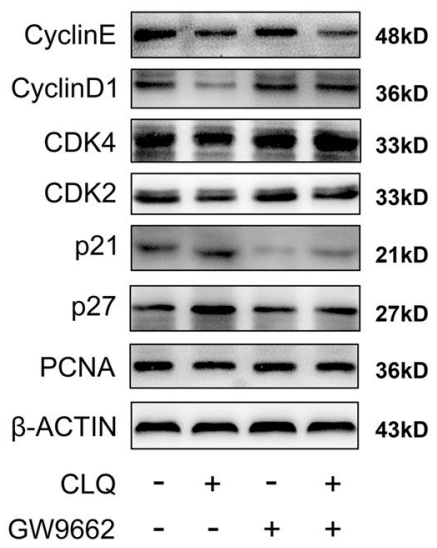

D

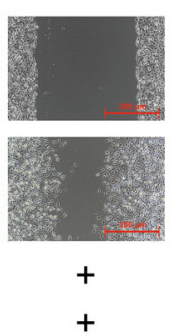

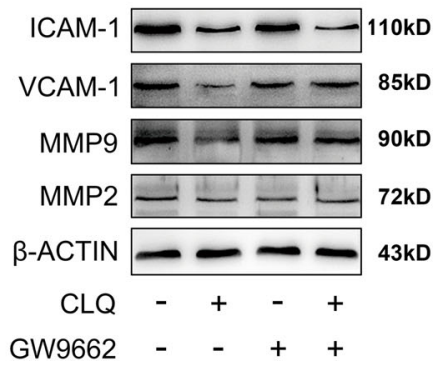

Fig. 6 PPARy antagonist alleviates the antimelanoma effects of CLQ in B16F10 cells. B16F10 cells were treated with $1.5 \mu \mathrm{M}$ CLQ with/without 10 MM GW9662 for $24 \mathrm{~h}$. a EdU incorporation assay. b Protein expression levels of key regulators involved in cell cycle progression. c Wound-healing assays. $\mathbf{d}$ Protein expression levels of ICAM-1, VCAM-1 and MMPs. All values are presented as the mean \pm SD from at least three separate experiments

Taken together, these data suggest that the inhibitory effects of CLQ on melanoma cell proliferation and migration are related to the suppression of the whole cell cycle and the hallmark protein expression involved in these processes.

Cancer cell proliferation and metastasis are closely associated with changes in glucose metabolism in tumors. Cancer cells exhibit high rates of aerobic glycolysis, a phenomenon historically known as the "Warburg effect" ${ }^{23,24}$. Accordingly, glucose uptake in these cells is increased to ensure the energy supply to compensate for the shortage of ATP because of glycolysis, leading to an excessive generation of lactate ${ }^{23,24}$. At the molecular level, GLUT1 contributes to the increased glucose utilization ${ }^{25}$. Next, HK2 catalyzes the first step of glucose metabolism, phosphorylating glucose to glucose-6-phosphate (G6P), while PKM2 switches the G6P into the glycolytic intermediate, pyruvate, which is converted into lactate by $\mathrm{LDHA}^{26}$. Remarkably, the inhibition of glycolysis suppresses tumor cell proliferation and migration ${ }^{27}$. For example, the inhibition of HK diminishes proliferation of cancer cells by decreasing the release of signaling proteins $^{28}$. The present study showed that CLQ exerted its antimelanoma effect by decreasing glucose consumption as well as ATP and lactate production by B16F10 cells. In addition, CLQ induced a major reduction of glycolysis in B16F10 cells. Consistently, CLQ significantly reduced the expression of glycolytic genes at both transcriptional and translational levels. To dissect the potential role of glycolysis in CLQ-induced impairment of B16F10 growth and metastasis, 2-DG, a competitive inhibitor of glucose transporters, was used to block the glycolysis in vivo and in vitro. We found that while CLQ and 2-DG alone suppressed the growth and metastasis of B16F10 cells as expected, the combination of these two reagents did not lead to a further inhibition both in vitro and in vivo, implicating that inhibition of glycolysis is potentially involved in mediating the antimelanoma actions of CLQ. Further studies would be necessary to fully elucidate the role of glycolysis in mediating CLQ's effects under the condition of the glycolysis inhibition and enhancement. Of note, in addition to its antitumor effects in melanoma, CLQ may also have a great potential in triggering a similar reduction of glycolysis in other cancer cells. Lastly, CLQ is also a mitochondrial uncoupler that increases oxygen consumption rate (OCR) in the presence of oligomycin in mouse cardiomyocytes. Hence, we hypothesized that CLQ may switch glycolysis to OXPHOS in B16F10 cells, which should be identified in the further study. 
A

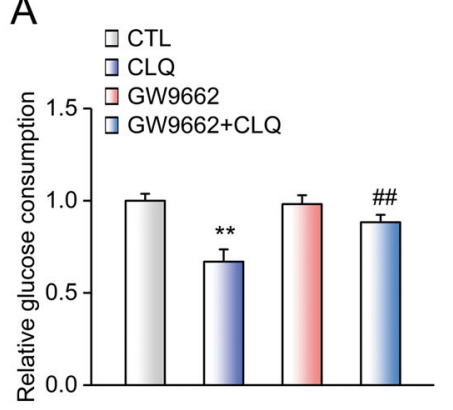

D

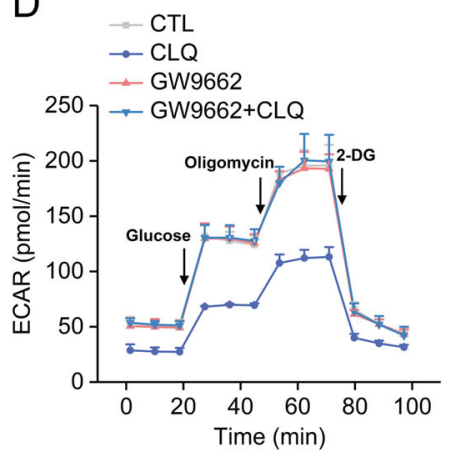

B

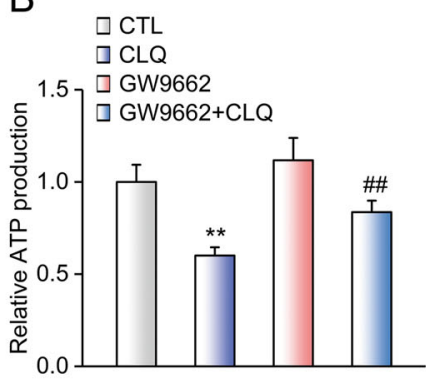

$\mathrm{E}$

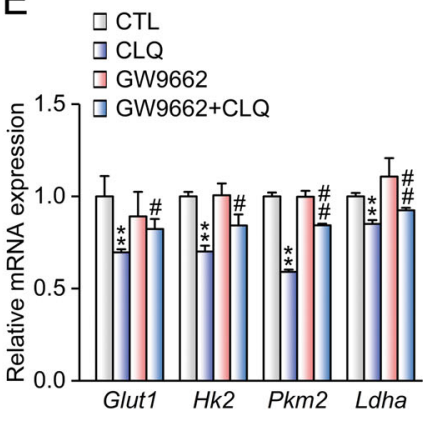

C

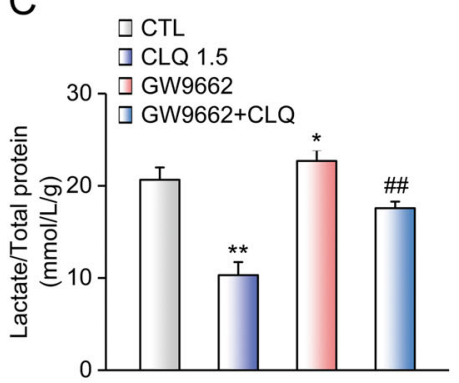

F

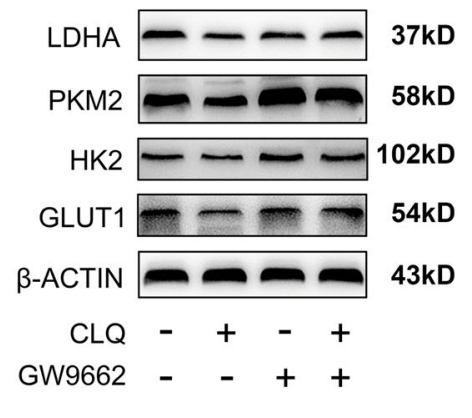

Fig. 7 PPAR $y$ mediates the inhibitory effects of CLQ on glycolysis in B16F10 cells. B16F10 cells were treated with $1.5 \mu M$ CLQ with/without $10 \mu \mathrm{M}$ GW9662 for $24 \mathrm{~h}$. a Glucose consumption. b Relative ATP production. c Lactate production. d ECAR. e RT-qPCR and (f) Western blot analyses of GLUT1, HK2, PKM2, LDHA expression levels. All values are presented as the mean \pm SD from at least three separate experiments. ${ }^{*} P<0.05$ and ${ }^{* *} P<$ 0.01 vs. CTL, ${ }^{\#} P<0.05$ and ${ }^{\# \#} P<0.01$ vs. CLQ

PPARY is a versatile transcriptional regulator involved in many pathophysiological processes, including adipocyte differentiation and glucose metabolism ${ }^{29,30}$. However, the role of PPARY in cancer is controversial, with studies showing either pro- or anti-neoplastic effects ${ }^{31-33}$. One possible explanation of this debate is that activation of PPARy induces growth inhibition in epithelial cancer cells, whereas it triggers tumor growth in stromal cells, indicating that the consequences of PPAR $\gamma$ activation for tumorigenesis are dependent on the cellular compartment $^{34}$. It is known that melanoma originates from the skin and exhibits an epithelial-like phenotype ${ }^{35}$. Hence, the activation of PPARY in B16F10 cells may lead to growth inhibition. Consistently, treatment with PPAR agonists dramatically blocks the proliferation of melanoma cells ${ }^{36}$. In the present study, we found that CLQ activated the PPAR transcription and increased PPAR $\gamma$ nuclear contents. Also, the extents of increases in PPAR $\gamma$ expression and nuclear fractions are quite comparable. These data suggest that CLQ increases PPARY activity initially at the transcriptional level, and the increase of PPARY nuclear contents is only a consequent event. Moreover, the PPAR $\gamma$-specific antagonist GW9662 or PPAR $\gamma$ shRNA partially abolished the antimelanoma effects of CLQ. These results indicated that PPAR $\gamma$ might serve as a potential molecular target of CLQ. On the other hand, CLQ could enhance the p53 activity to switch its pro-apoptotic ability into a protective response including activation of the $\mathrm{p} 21$ expression ${ }^{15}$. In our study, we found that CLQ increases the protein expression levels of $\mathrm{p} 21$ but does not alter p53 levels, indicating that the inhibitory effects of CLQ on melanoma are p53-independent.

PPAR $\gamma$ agonists, such as rosiglitazone and pioglitazone, have been widely used in the clinical treatment of various diseases, including type 2 diabetes ${ }^{37}$. Although these thiazolidinediones (TZDs) exhibit satisfactory effects on improving insulin sensitivity and hyperglycemia, most of them have detrimental side effects ${ }^{38}$. For example, troglitazone was withdrawn because of liver toxicity ${ }^{39}$. In addition, administration of rosiglitazone is tightly correlated with an increased risk of cardiovascular diseases in patients, while pioglitazone has been associated with an increased fracture risk $^{40,41}$. These limitations aroused substantial concerns and significantly dampened TZD's future in many countries. Therefore, it is critical to develop TZD substitutes or non-TZD selective PPAR $\gamma$ agonists for improved therapies of diseases associated with PPAR $\gamma$ activity. In the present study, we found that CLQ increased the PPARY transcription and that such activation did not cause any liver toxicity in mice. Hence, 


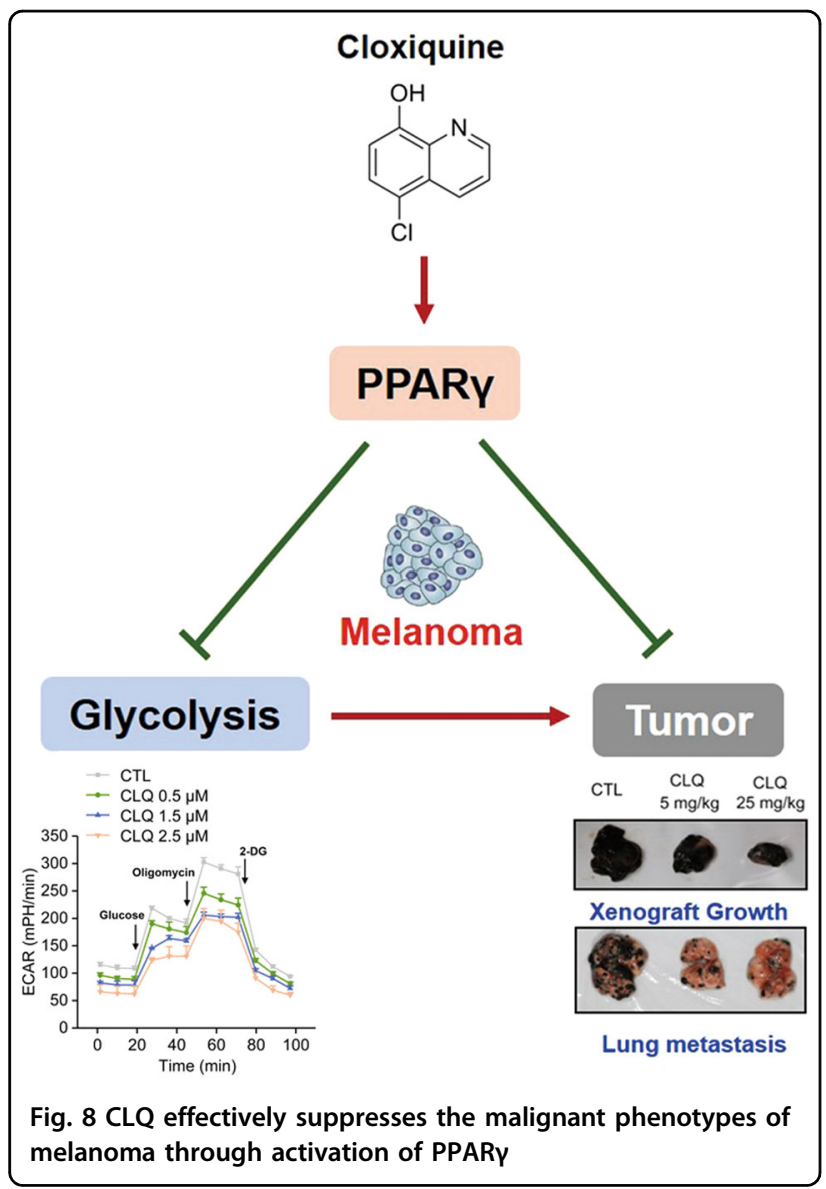

as an FDA-approved clinical drug, CLQ may be a new non-TZD selective PPARY agonist and further evidence should be provided to establish whether CLQ performs a similar function in the treatment of metabolic diseases, including insulin resistance and hyperglycemia.

In conclusion, our findings demonstrated the potential use of CLQ in reducing glycolysis and inhibiting proliferation and metastasis of melanoma cells. Mechanistically, PPAR $\gamma$ plays a nonredundant role in mediating these beneficial effects (Fig. 8). These results imply that in addition to its antituberculosis use, CLQ is a promising candidate in the therapy of melanoma cancer progression.

\section{Materials and methods Cell culture}

All cell lines were obtained from the American Type Culture Collection (ATCC) and grown at $37^{\circ} \mathrm{C}$ in $5 \%$ $\mathrm{CO}_{2}-95 \%$ air. B16F10, A375 and PIG1 cells were cultured in DMEM supplemented with $10 \%$ fetal bovine serum (FBS, Sciencell Research, Carlsbad, CA, USA) and 1\% antibiotics (penicillin and streptomycin). Melan-A cells were grown in RPMI-1640 medium containing 10\% FBS and $1 \%$ antibiotics.

\section{Drug screening assay}

CCK-8 assay was used to screen functional drugs that could suppress the proliferation of melanoma cells. In brief, $5 \times 10^{3}$ cells were seeded into each well of a 96-well plate and cultured at $37^{\circ} \mathrm{C}$ overnight. After synchronization with serum-free DMEM, cells were transferred into $100 \mu \mathrm{L}$ of serum-free DMEM containing either $10 \mu \mathrm{M}$ drugs (1430 small compounds) or vehicle (0.1\% DMSO) and incubated for another $24 \mathrm{~h}$. Then, $10 \mu \mathrm{L}$ of WST-8 reagent (Jiancheng, Nanjing, Jiangsu, China) was added to each well and incubated at $37^{\circ} \mathrm{C}$ for $2 \mathrm{~h}$. Finally, a microplate reader was used to measure the absorbance at $450 \mathrm{~nm}$. An inhibition efficiency of cell viability more than $75 \%$ were considered significant.

\section{Morphometric analysis}

All cell lines were treated with CLQ for $24 \mathrm{~h}$ and then fixed in $4 \%$ paraformaldehyde solution for $30 \mathrm{~min}$. Cell morphologies were photographed with a Nikon microscope (ECLIPSE, Ts2R-FL, Tokyo, Japan).

\section{Proliferation and migration assays}

Cell proliferation was analyzed by using an EdU incorporation assay, while cell migration was detected by using wound healing and transwell chamber assays. These experiments were performed as described previously ${ }^{17}$.

\section{RT-qPCR and Western blot}

Total RNA from B16F10 cells were extracted using Trizol reagent (Invitrogen, Carlsbad, CA, USA). For mRNA detection, $1 \mu \mathrm{g}$ total RNA was reverse-transcribed into complementary DNA. Real-time PCR amplification was performed using SYBR premix Ex Taq (Vazyme, Nanjing, Jiangsu, China) and the LightCycler ${ }^{\oplus} 480$ System (Roche, Basal, Switzerland). The primers for mouse 36B4 were included for normalization. A complete list of PCR primers is shown in Supplementary Table 4. For protein analysis, cells were lysed in RIPA buffer. The protein concentration was quantified with a BCA protein quantification kit (Jiancheng, Nanjing, Jiangsu, China). Equal amounts of protein were loaded and separated by $10 \%$ SDS-PAGE and then transferred onto PVDF membranes (Millipore, Bedford, MA, USA). The membranes were incubated overnight with appropriate primary antibodies. HRP-conjugated secondary antibodies were then applied to bind and visualize the primary antibodies. Quantitative analysis was performed by NIH ImageJ $1.32 \mathrm{j}$ software. To detect the translocation of PPAR $\gamma$ protein to the nuclei, we extracted nuclear protein using a commercial kit (Shengxing, Shanghai, China) and then performed Western blot analysis. Histone $\mathrm{H} 3$ was used as the loading control. Antibodies against Cyclin E (Cat. No. 4129; 1:1000 dilution), PCNA (Cat. No. 2586; 1:1000 dilution), CDK2 (Cat. No. 2546; 1:1000 dilution), Cyclin D1 (Cat. 
No. 2922; 1:1000 dilution), p27 (Cat. No. 3686; 1:1000 dilution) and PPARY (Cat. No. 2443 S; 1:1000 dilution) were purchased from Cell Signaling Technology (Danvers, MA, USA). Antibodies against CDK4 (Cat. No. 610147; 1:1000 dilution) and p21 (Cat. No. 556430; 1:1000 dilution) were purchased from BD Bioscience (San Jose, CA, USA). Antibodies against ICAM-1 (Cat. No. sc-1511; 1:500 dilution), VCAM-1 (Cat. No. sc-1504; 1:500 dilution), MMP-2 (Cat. No. sc-10736; 1:500 dilution) and MMP-9 (Cat. No. sc-6840; 1:500 dilution) were purchased from Santa Cruz (Dallas, TX, USA). Antibodies against LDHA (Cat. No. 19987; 1:1000 dilution), PKM2 (Cat. No. 15822; 1:1000 dilution), HK2 (Cat. No. 13938; 1:1000 dilution), GLUT1 (Cat. No. 21829; 1:1000 dilution) and p53 (Cat. No. 10442; 1:1000 dilution) were purchased from Proteintech (Chicago, IL, USA). Antibodies against Histone H3 (Cat. No. BS1174; 1:1000 dilution) and $\beta$-ACTIN (Cat. No. BS6007MH; 1:1000 dilution) were purchased from Bioworld Technology (Nanjing, Jiangsu, China).

\section{Animals}

All animal procedures in this investigation conform to the Guide for the Care and Use of Laboratory Animals published by the US National Institutes of Health (NIH publication No. 85-23, revised 1996) and the approved regulations set by the Laboratory Animal Care Committee at China Pharmaceutical University (Permit number SYXK-2016-0011). All mice were maintained in a $12 \mathrm{~h}$ light $/ 12 \mathrm{~h}$ dark cycle and a temperature- and humidity-controlled environment. To detect the effect of tumor growth in vivo, mouse B16F10 cells $\left(1 \times 10^{6}\right.$ cells per mouse) were injected subcutaneously into the right flanks of nude mice (Model Animal Research Center of Nanjing University, Nanjing, Jiangsu, China). Tumors were allowed to grow for seven days, and then mice were administered vehicle (olive, intraperitoneally (i.p.), daily) or CLQ ( $5 \mathrm{mg} / \mathrm{kg}, 25 \mathrm{mg} / \mathrm{kg}$, i.p., daily) for eight days. The dose of CLQ was chosen based on a previous study showing that this CLQ dose functionally protected from radiation-induced gastrointestinal death in mice ${ }^{15}$. For the metastasis analysis, mouse B16F10 cells $\left(1.5 \times 10^{5}\right.$ cells per mouse) were injected intravenously. Seven days later, mice were randomly divided into three groups and were administered vehicle (olive, i.p., daily) or CLQ ( $5 \mathrm{mg} / \mathrm{kg}, 25 \mathrm{mg} / \mathrm{kg}$, i.p., daily) for fourteen days. Then, mice were euthanized, their lungs were removed and fixed, and the number of macroscopic black metastases on the surfaces of the lungs were enumerated. To detect whether glycolysis mediates the antimelanoma effects of CLQ, 2-DG was i.p. injected into mice at a dose of $500 \mathrm{mg} / \mathrm{kg}$ body weight once every other day in addition to CLQ ( $25 \mathrm{mg} / \mathrm{kg}$, i.p., daily) administration.

\section{IHC and TUNEL assays}

All specimens were isolated, fixed in $4 \%$ paraformaldehyde solution for $24 \mathrm{~h}$ in situ, processed for paraffin embedding, and cut into $4 \mu \mathrm{m}$ transverse sections for routine $\mathrm{H} \& \mathrm{E}$ staining. For IHC analysis, tumor sections were incubated with antibody against mouse Ki-67 (Cat. No. 12202; 1:200 dilution, Cell Signaling Technology) at $4{ }^{\circ} \mathrm{C}$ overnight for the later immunostaining by using diaminobenzidine (DAB). For TUNEL analysis, liver sections were incubated with TUNEL labeling (marked with Green fluorescence) mix at $37^{\circ} \mathrm{C}$ for $60 \mathrm{~min}$, and then double stained with DAPI (marked with Blue fluorescence, Sigma, USA). The sections were photographed with a Nikon fluorescence microscope (ECLIPSE, Ts2RFL, Tokyo, Japan). The slides were scanned by a Pannoramic Flash 250 scanner (Perkin Elmer, Waltham, MA, USA) and viewed by the Pannoramic viewer software program (3D Histech, Waltham, MA, USA). Quantitative analysis was performed by NIH ImageJ 1.32j software.

\section{Serological analysis}

Blood samples were collected in nonheparinized tubes and centrifuged at 4000 r.p.m. for $10 \mathrm{~min}$ at $4{ }^{\circ} \mathrm{C}$. The serum levels of ALT and AST were determined spectrophotometrically using commercial kits (Jiancheng Institute of Biotechnology, Nanjing, Jiangsu, China).

\section{Glucose consumption, ATP and lactate production}

Mouse B16F10 cells were treated with indicated doses of CLQ with/without GW9662 (10 $\mu \mathrm{M}$, PPAR $\gamma$ antagonist) for $24 \mathrm{~h}$. The concentration of glucose in culture medium was measured by the Glucose Test Kit (Rongsheng Biotechnology, Shanghai, China). The intracellular concentrations of lactate and ATP were determined by using commercial kits (Sigma, St Louis, MO, USA for lactate, and Promega, Madison, WI, USA for ATP), according to the manufacturer's instructions.

\section{ECAR assay}

The ECAR was analyzed using a Seahorse XF24 Extracellular Flux Analyzer (Seahorse Bioscience, North Billerica, MA, USA). In brief, $2 \times 10^{5}$ cells were seeded into each well of a Seahorse XF24 cell culture microplate. On the next day, the cells were treated with/without CLQ at indicated doses for $24 \mathrm{~h}$, and GW9662 was added when necessary. Then, cells were switched to a basal glucosefree medium for $1 \mathrm{~h}$ prior to measurement. A default standard glycolysis stress-test program was performed. During measurement, glucose (final concentration $10 \mathrm{mM}$ ), oligomycin (final concentration $1 \mu \mathrm{M}$ ) and 2-DG (final concentration $100 \mathrm{mM}$ ) were sequentially injected into each well at the indicated time points. Data were derived from Seahorse XF24 Wave software and displayed in $\mathrm{pmol} / \mathrm{minute}$. 


\section{Reverse pharmacophore screening}

To predict the potential molecular target of CLQ, we performed PharmMapper (http://lilab.ecust.edu.cn/ pharmmapper/) and DRAR-CPI (http://cpi.bio-x.cn/drar/) analyses. PharmMapper is a web-based tool that predicts the binding of a small molecule to potential targets via a "reverse" pharmacophore mapping approach ${ }^{42}$. All the predicted targets were screened and sorted by Normalized Fit-score in a descending order. DRAR-CPI is a server that is used for repositioning drugs based on the analysis of the chemical-protein interactome ${ }^{43}$. All DRAR-CPI-calculated results were ordered by the default index Z'-score in ascending order. After prediction, the top 10 candidates were selected and clustered for their potential targets.

\section{ICC assay}

To analyze the PPARY intracellular localization, mouse B16F10 cells were treated with or without CLQ $(1.5 \mu \mathrm{M}$, an effective dose in inhibiting proliferation, migration, and glycolysis in B16F10 cells) for $24 \mathrm{~h}$. After that, cells were fixed with ice-cold $4 \%$ paraformaldehyde for $30 \mathrm{~min}$, followed by blocking in $5 \%$ goat serum for $1 \mathrm{~h}$, and incubation with rabbit polyclonal anti-PPAR $\gamma$ antibody overnight at $4{ }^{\circ} \mathrm{C}$. After repeated washing, the cells were probed with secondary antibodies conjugated to Alexa Fluor 488 anti-Rabbit IgG (Cat. No. 4408 S, 1:500 dilution, Cell Signaling Technology) for $1 \mathrm{~h}$ at room temperature. Nuclei were identified with DAPI. The sections were photographed with a Nikon fluorescence microscope (ECLIPSE, Ts2R-FL, Tokyo, Japan).

\section{PPARY ShRNA transfection}

Mouse PPAR $\gamma$ shRNA (5'-CCATCCGATTGAAGCTTATTTATGA-3') and non-specific shRNA (Scramble shRNA, 5'-TTCTCCGAACGTGTCACGTAA-3') were designed and synthesized by GenePharma (Shanghai, China). To knockdown the expression of PPAR $\gamma$ in B16F10 cells, the cells were transfected with scramble shRNA or PPARY shRNA $24 \mathrm{~h}$ in advance of indicated treatments. Cell transfection were performed by using Lipofectamine 3000 reagent (Invitrogen, Carlsbad, CA, USA) according to the manufacturer's instructions.

\section{Statistical analysis}

Statistical analysis was performed by using the Origin 8 software (version 8.6, OriginLab Corporation, USA). Groups of data were presented as the mean \pm SD (standard deviation). One-way ANOVA followed by Fisher's LSD post hoc test were performed to analyze the data. A value of $P<0.05$ was considered as statistically significant.

\section{Acknowledgements}

This work was financially supported by grants from the National Natural Science Foundation of China $(31800992,81800512,31771298)$, the Natural
Science Foundation of the Jiangsu Higher Education Institutions of China (BK20180554, BK20180577), the Project of State Key Laboratory of Natural Medicines, China Pharmaceutical University (SKLNMZZRC201803), the "Double First-Class" University Project (CPU2018GY17, CPU2018GY18), the Fundamental Research Funds for the Central Universities (2632018PY15), the Open Fund of State Key Laboratory of Pharmaceutical Biotechnology, Nanjing University, China (KF-GN-201901).

\section{Author details}

${ }^{1}$ State Key Laboratory of Natural Medicines, China Pharmaceutical University, Nanjing, Jiangsu, China. ${ }^{2}$ School of Life Science and Technology, China Pharmaceutical University, Nanjing, Jiangsu, China. ${ }^{3}$ State key Laboratory of Pharmaceutical Biotechnology, Nanjing University, Nanjing, Jiangsu, China

\section{Conflict of interest}

The authors declare that they have no conflict of interest.

\section{Publisher's note}

Springer Nature remains neutral with regard to jurisdictional claims in published maps and institutional affiliations.

Supplementary Information accompanies this paper at (https://doi.org/ 10.1038/s41419-019-1644-8).

Received: 15 January 2019 Revised: 10 May 2019 Accepted: 15 May 2019 Published online: 28 May 2019

\section{References}

1. Govindarajan, B. et al. Malignant transformation of melanocytes to melanoma by constitutive activation of mitogen-activated protein kinase kinase (MAPKK) signaling. J Biol Chem. 278, 9790-9795 (2003).

2. Bittner, M. et al. Molecular classification of cutaneous malignant melanoma by gene expression profiling. Nature 406, 536-540 (2000).

3. Tucker, M. A. \& Goldstein, A. M. Melanoma etiology: where are we? Oncogene 22, 3042-3052 (2003).

4. Diepgen, T. L. \& Mahler, V. The epidemiology of skin cancer. Br. J. Dermatol. 146(Suppl 61), 1-6 (2002).

5. Siegel, R. L., Miller, K. D. \& Jemal, A. Cancer Statistics, 2017. CA Cancer J. Clin. 67, 7-30 (2017).

6. Glazer, A. M., Winkelmann, R. R., Farberg, A. S. \& Rigel, D. S. Analysis of trends in US melanoma incidence and mortality. JAMA Dermatol. 153, 225-226 (2016).

7. Fang, M. et al. In vitro and in vivo anti-malignant melanoma activity of Alocasia cucullata via modulation of the phosphatase and tensin homolog/phosphoinositide 3-kinase/AKT pathway. J. Ethnopharmacol. 213, 359-365 (2018).

8. Florea, A. M. \& Busselberg, D. Cisplatin as an anti-tumor drug: cellular mechanisms of activity, drug resistance and induced side effects. Cancers $\mathbf{3}$, 1351-1371 (2011).

9. Kehe, K. \& Szinicz, L. Medical aspects of sulphur mustard poisoning. Toxicology 214, 198-209 (2005).

10. Homet, B. \& Ribas, A. New drug targets in metastatic melanoma. J. Pathol. 232, 134-141 (2014).

11. Hodi, F. S. et al. Improved sunvival with ipilimumab in patients with metastatic melanoma. N Engl. J. Med. 363, 711-723 (2010).

12. Postow, M. A. et al. Nivolumab and ipilimumab versus ipilimumab in untreated melanoma. N Engl J. Med. 372, 2006-2017 (2015).

13. Ashburn, T. T. \& Thor, K. B. Drug repositioning: identifying and developing new uses for existing drugs. Nat. Rev. Drug Discov. 3, 673-683 (2004).

14. Zakikhani, M., Dowling, R., Fantus, I. G., Sonenberg, N. \& Pollak, M. Metformin is an AMP kinase-dependent growth inhibitor for breast cancer cells. Cancer Res. 66, 10269-10273 (2006).

15. Morita, A. et al. A chemical modulator of p53 transactivation that acts as a radioprotective agonist. Mol. Cancer Ther. 17, 432-442 (2018).

16. Zhang, J., Nadtochiy, S. M., Urciuoli, W. R. \& Brookes, P. S. The cardioprotective compound cloxyquin uncouples mitochondria and induces autophagy. Am. J. Physiol. Heart Circ. Physiol. 310, H29-H38 (2016). 
17. Chen, S., Ding, Y., Zhang, Z., Wang, H. \& Liu, C. Hyperlipidaemia impairs the circadian clock and physiological homeostasis of vascular smooth muscle cells via the suppression of Smarcd1. J. Pathol. 233, 159-169 (2014).

18. Hanahan, D. \& Weinberg, R. A. Hallmarks of cancer: the next generation. Cell 144, 646-674 (2011)

19. Vander Heiden, M. G., Cantley, L. C. \& Thompson, C. B. Understanding the Warburg effect: the metabolic requirements of cell proliferation. Science 324, 1029-1033 (2009).

20. Latosinska, J. N., Latosinska, M., Tomczak, M. A., Seliger, J. \& Zagar, V. Supramolecular synthon pattern in solid clioquinol and cloxiquine (APIs of antibacterial, antifungal, antiaging and antituberculosis drugs) studied by (3)(5)Cl NQR, (1)H-(1)(7)O and (1)H-(1)(4)N NQDR and DFT/QTAIM. J. Mol. Model 17 1781-1800 (2011).

21. Malumbres, M. \& Barbacid, M. Cell cycle, CDKs and cancer: a changing paradigm. Nat. Rev. Cancer 9, 153-166 (2009).

22. Bhatia, S., Tykodi, S. S. \& Thompson, J. A. Treatment of metastatic melanoma: an overview. Oncology 23, 488-496 (2009).

23. Hsu, P. P. \& Sabatini, D. M. Cancer cell metabolism: Warburg and beyond. Cell 134, 703-707 (2008).

24. Pelicano, H., Martin, D. S., Xu, R. H. \& Huang, P. Glycolysis inhibition for anticancer treatment. Oncogene 25, 4633-4646 (2006).

25. Szablewski, L. Expression of glucose transporters in cancers. Biochim. Biophys. Acta 1835, 164-169 (2013).

26. Cairns, R. A., Harris, I. S. \& Mak, T. W. Regulation of cancer cell metabolism. Nat. Rev. Cancer 11, 85-95 (2011).

27. Gao, C. et al. Jolkinolide B induces apoptosis and inhibits tumor growth in mouse melanoma B16F10 cells by altering glycolysis. Sci. Rep. 6, 36114 (2016).

28. Gottlob, K. et al. Inhibition of early apoptotic events by Akt/PKB is dependent on the first committed step of glycolysis and mitochondrial hexokinase. Genes Dev. 15, 1406-1418 (2001).

29. Rosen, E. D. et al. PPAR gamma is required for the differentiation of adipose tissue in vivo and in vitro. Mol. Cell 4, 611-617 (1999).

30. Martin, G., Schoonjans, K., Staels, B. \& Auwerx, J. PPARgamma activators improve glucose homeostasis by stimulating fatty acid uptake in the adipocytes. Atherosclerosis 137(Suppl), S75-S80 (1998).
31. Lefebvre, A. M. et al. Activation of the peroxisome proliferator-activated receptor gamma promotes the development of colon tumors in C57BL/6JAPCMin/ + mice. Nat. Med. 4, 1053-1057 (1998).

32. Panasyuk, G. et al. PPARgamma contributes to PKM2 and HK2 expression in fatty liver. Nat. Commun. 3, 672 (2012).

33. Elstner, E. et al. Ligands for peroxisome proliferator-activated receptorgamma and retinoic acid receptor inhibit growth and induce apoptosis of human breast cancer cells in vitro and in BNX mice. Proc. Natl Acad. Sci. USA 95, 8806-8811 (1998).

34. Avena, P. et al. Compartment-specific activation of PPARgamma governs breast cancer tumor growth, via metabolic reprogramming and symbiosis. Cell Cycle 12, 1360-1370 (2013).

35. Li, F. Z., Dhillon, A. S., Anderson, R. L., McArthur, G. \& Ferrao, P. T. Phenotype switching in melanoma: implications for progression and therapy. Front. Oncol. 5, 31 (2015).

36. Liu, Y. et al. Growth inhibition and differentiation induced by peroxisome proliferator activated receptor gamma ligand rosiglitazone in human melanoma cell line A375. Med. Oncol. 23, 393-402 (2006).

37. Kersten, S., Desvergne, B. \& Wahli, W. Roles of PPARs in health and disease Nature 405, 421-424 (2000).

38. Shah, P. \& Mudaliar, S. Pioglitazone: side effect and safety profile. Expert Opin. Drug Saf. 9, 347-354 (2010).

39. Watkins, P. B. Insight into hepatotoxicity: The troglitazone experience. Hepatology 41, 229-230 (2005).

40. Singh, S., Loke, Y. K. \& Furberg, C. D. Long-term risk of cardiovascular events with rosiglitazone: a meta-analysis. JAMA 298, 1189-1195 (2007).

41. Aubert, R. E., Herrera, V., Chen, W., Haffner, S. M. \& Pendergrass, M. Rosiglitazone and pioglitazone increase fracture risk in women and men with type 2 diabetes. Diabetes Obes. Metab. 12, 716-721 (2010).

42. Liu, X. et al. PharmMapper server: a web server for potential drug target identification using pharmacophore mapping approach. Nucleic Acids Res. 38 W609-W614 (2010).

43. Luo, $\mathrm{H}$. et al. DRAR-CPI: a server for identifying drug repositioning potential and adverse drug reactions via the chemical-protein interactome. Nucleic Acids Res. 39, W492-W498 (2011). 